\title{
CAPÍTULO 44: REVESTIMENTOS UTILIZADOS EM NANOPARTÍCULAS MAGNÉTICAS PARA IMOBILIZAÇÃO DE ENZIMAS: UMA REVISÃO
}

\section{CHAPTER 44: COATINGS USED IN MAGNETIC NANOPARTICLES FOR ENZYME IMMOBILIZATION: A REVISION}

\author{
Munique Cristiane Tavares Santos Silva ${ }^{1}$; Lígia Maria Gonçalves Fernandes ${ }^{2}$; Ana Lúcia Figueiredo \\ Porto $^{3}$; Márcia Nieves Carneiro da Cunha ${ }^{4}$; Tatiana Souza Porto ${ }^{5}$
}

\begin{abstract}
Resumo
Enzimas imobilizadas em nanopartículas magnéticas (NPMs) possuem ampla aplicabilidade em processos catalíticos industriais, devido a vantagens como: aumento da produtividade e da estabilidade operacional, facilidade de manuseio e separação, além da possibilidade de reutilização do biocatalizador. Entretanto, a imobilização direta de enzimas na superfície dessas partículas não é recomendada, em razão de sua fraca dispersibilidade aquosa e alta tendência a oxidação. Visando contornar este problema, as NPMs são geralmente revestidas com materiais inertes e impermeáveis. Desta forma, este trabalho teve como objetivo realizar uma breve busca na literatura sobre os materiais comumente utilizados para revestimento de nanopartículas magnéticas. A escolha adequada da natureza e do tipo de material utilizado possibilita o aprimoramento no processo de imobilização, além de aumentar a estabilidade e eficiência catalítica da enzima.
\end{abstract}

Palavras-Chave: imobilização enzimática, nanopartículas magnéticas, revestimento.

\begin{abstract}
Enzymes immobilized in magnetic nanoparticles (MNPs) have wide applicability in industrial catalytic processes, due to advantages such as: increased productivity and operational stability, ease of handling and separation, in addition to the possibility of biocatalyst reusing. However, direct immobilization of enzymes on the surface of these particles is not recommended, due to their poor aqueous dispersibility and high tendency to oxidation. To circumvent this problem, MNPs are generally coated with inert and impermeable materials. Thus, this work aimed to conduct a brief search in the literature on the materials commonly used for coating magnetic nanoparticles. The appropriate choice of the nature and type of material used makes it possible to improve the immobilization process, in addition to increasing stability and catalytic efficiency.
\end{abstract}

Keywords: enzymatic immobilization, magnetic nanoparticles, coating.

\footnotetext{
1 Programa de Pós Graduação em Biociência Animal, Universidade Federal Rural de Pernambuco, muniquecpc@gmail.com

${ }^{2}$ Programa de Pós Graduação em Biociência Animal, Universidade Federal Rural de Pernambuco, proflmgf@yahoo.com.br

3 Departamento de Morfologia e Fisiologia Animal, Universidade Federal Rural de Pernambuco, analuporto@yahoo.com.br

4 Departamento de Morfologia e Fisiologia Animal, Universidade Federal Rural de Pernambuco, marcianieves@yahoo.com.br

5 Doutora em Tecnologia Bioquímico-Farmacêutica, Universidade Federal do Agreste de Pernambuco, tatiana.porto@ufape.edu.br
} 


\section{Introdução}

Enzimas são biocatalizadores altamente específicos, que podem ter seu uso maximizado e melhorado através de sua imobilização. Este processo pode permitir à enzima se tornar mais estável e resistente a condições severas, como grandes intervalos de $\mathrm{pH}$ e temperatura, e também pode preservar atividade enzimática por de vários ciclos. A imobilização enzimática é uma alternativa à aplicação eficiente destes biocatalisadores nas indústrias alimentícia, química e farmacêutica. Para que o uso de enzimas imobilizadas seja economicamente viável, deve-se considerar o tipo de suporte e o método de imobilização que será empregado, visto que este irá influenciar na atividade enzimática e na posterior reutilização desses biocatalizadores (TOMBÁCZ et al., 2015).

Uma das grandes questões do processo de imobilização enzimática é a escolha do suporte, o uso de um suporte adequado é essencial para definir o tipo de imobilização a ser realizado, uma vez que o material utilizado poderá afetar significativamente as propriedades da enzima imobilizada. Os suportes podem ser orgânicos ou inorgânicos e devem ser inertes, estáveis, insolúveis no meio de reação e resistentes às forças mecânicas (GENNARI et al., 2020). O uso de suportes com núcleo magnético diminui a necessidade de centrifugação, a indesejável diluição da amostra e perdas do suporte durante as lavagens, eventos que muitas vezes complicam o uso de reatores de enzimas não-magnéticas. As separações magnéticas são relativamente rápidas, fáceis e requerem aparelhagem simples. Portanto, técnicas de separação magnética têm atualmente encontrado muitas aplicações em áreas diferentes das ciências biológicas, especialmente em escala laboratorial (SEENUVASAN, 2020)

Nanopartículas magnéticas possuem elementos magnéticos que podem ser manipulados por um campo externo, apresentam tamanhos que variam de 1 à $100 \mathrm{~nm}$, tem superfície hidrofílica e alta razão superfície/volume, podem estar em estado superparamagnético, entre outras características físicas e químicas que são fortemente influenciadas pelo seu tamanho, morfologia e estrutura cristalina (BEDÊ, 2010).

Entretanto, nanopartículas magnéticas apresentam certas limitações práticas quando utilizadas em aplicações biológicas e farmacológicas. Dentre esses empecilhos, destacam-se a tendência de se aglomerarem a fim de minimizar a energia livre superficial; baixas estabilidade e dispersividade em determinados solventes (em especial, na água); alta atividade química; facilidade em ser oxidada em ar (principalmente a magnetita) e, consequente perda de propriedades magnéticas; absorção pelo corpo; e biodegradação em meio biológico, ocasionando perda de suas propriedades originais (WU, HE e JIANG, 2008). Dessa forma, proporcionar um 
recobrimento apropriado dessas partículas mostra-se como uma boa alternativa para amenizar e/ou eliminar essas barreiras, e proporcionar maior eficiência de imobilização enzimática. Diante do exposto, este trabalho objetivou realizar uma busca bibliográfica a respeito dos diversos revestimentos utilizados em nanopartículas magnéticas, avaliando a eficiência deste revestimento na imobilização, na estabilidade e na capacidade de reutilização da enzima imobilizada.

\section{Desenvolvimento}

\section{Enzimas}

Micro-organismos são fontes atrativas para obtenção de enzimas de uso industrial, pois apresentam grande disponibilidade, taxa de crescimento rápido e fácil manipulação genética, podendo ser cultivados em grandes quantidades sob condições controladas, em meios cultivo de baixo custo e elevada produtividade. Acrescenta-se ainda a vantagem de a produção não estar condicionada às questões sazonais e geográficas (BON et al., 2009). Aplicações de enzimas microbianas em indústrias alimentícias, farmacêuticas, têxteis, papel, couro e outras são numerosas e crescem rapidamente em relação aos métodos convencionais, devido a menos danos ao meio ambiente, maior eficiência e produtos de maior qualidade (SINGH et al., 2016).

Espera-se que o mercado global de enzimas tenha no período de 2019 a 2026 uma taxa anual de crescimento de 6,75\%, passando de U\$ 8,8 bilhões em 2018 para U\$ 13,79 bilhões até 2026. Este crescimento acelerado de aproximadamente $68 \%$ no período descrito é dado devido ao uso extensivo das enzimas em diversos setores da indústria, tais como, processamento de alimentos, papel, biocombustíveis, detergentes biológicos, setores de biologia molecular, entre outros (FIORMARKETS, 2019).

$\mathrm{Na}$ indústria farmacêutica e de diagnóstico, as aplicações enzimáticas estão crescendo rapidamente. Aplicações relacionadas com o debridamento de feridas por enzimas proteolíticas com atividade colagenolítica e o bloqueio de coágulos pelas enzimas fibrinolíticas são proeminentes (SINGH et al., 2016). Um exemplo disto é a protease fibrinolitica produzida por Mucor subtilissimus UCP1262 um promissor agente antitrombótico (NASCIMENTO et al., 2016).

O uso de enzimas ou micro-organismos em preparações alimentícias é um processo antigo. A biotecnologia de alimentos, considerada como aplicações de processos biotecnológicos na produção de alimentos, começou antes de 6000 a.C. Fermentar uvas ou fabricar cerveja são exemplos dessa biotecnologia alimentar inicial, apesar de serem rudimentares, a aplicação de enzimas na tecnologia de alimentos já foi estabelecida nesses processos (TAVANO et al., 2018). Com o avanço da tecnologia, 
novas enzimas foram desenvolvidas e novas áreas de aplicação ainda estão sendo exploradas.

As enzimas são utilizadas em praticamente todos os segmentos da indústria de alimentos (panificação, laticíneos, bebidas). Um exemplo disto são as pectinases ácidas que podem ser usadas na extração, clarificação e remoção de pectina dos sucos de frutas, a maceração de vegetais para produzir pastas purês e vinificação. Quase todas as preparações comerciais de pectinases são produzidas por espécies fúngicas, pertencentes principalmente ao gênero Aspergillus. Entre eles, Aspergillus aculeatus destaca-se na produção industrial de enzimas pectinolíticas, visto que a preparação comercial Pectinex ${ }^{\circledR}$ Ultra SPL, obtido a partir deste micro-organismo, foi empregado com sucesso para clarificar e reduzir a viscosidade de sucos de frutas, como maçã, carambola e pitaya vermelha (OLIVEIRA et al., 2018).

$\mathrm{Na}$ indústria têxtil e do couro, as enzimas são utilizadas em diferentes etapas do processo de produção industrial. Na indústria têxtil as enzimas vêm sendo utilizadas no desenvolvimento de processos visando minimizar a geração de resíduos e estratégias para melhorar a qualidade do produto final (CHOI et al., 2015). As principais classes de enzimas envolvidas nos processos de pré-tratamento e acabamento do algodão são as hidrolases e as oxidorredutases. O grupo das hidrolases incluem amilase, celulase, quitinase, protease, pectinase e lipase, que estão envolvidas no biopolimento e biossouramento de tecidos, antifelting de lã, amaciamento de algodão, acabamento de jeans, acabamento de lã e modificação de fibras sintéticas. As oxidorredutases incluem catalase, lacase, peroxidase e ligninase, que estão envolvidas no bio-branqueamento, na finalização do branqueamento, na descoloração do corante, no tecido e no acabamento da lã (SINGH et al., 2018).

As enzimas proteolíticas alcalinas são preferíveis aos sintéticos convencionais devido à sua especificidade de substrato, propriedades de limpeza, melhor desempenho em temperaturas de lavagem e atenuação da poluição ambiental. Essas características garantem alta aplicabilidade nas indústrias de detergentes como agente de limpeza e na indústria de cosméticos como sabonetes enzimáticos (FERNANDES et al., 2020).

\section{Imobilização de enzimas}

A utilização de enzimas em processos industriais requer o desenvolvimento de técnicas que possibilitem a sua recuperação e reutilização, visando tornar o processo economicamente viável, o que pode ser alcançado a partir da utilização de técnicas de imobilização. A imobilização enzimática favorece a estabilidade da enzima uma vez que o processo pode alterar suas propriedades estruturais, produzindo biocatalisadores com 
elevada atividade, especificidade e estabilidade, sendo por isso considerada, nos últimos anos, a técnica mais promissora para tornar competitiva a aplicação de enzimas em larga escala (SOUZA et al., 2017).

Imobilização é um termo genérico empregado para descrever a retenção de uma biomolécula no interior de um reator ou de um sistema analítico (CARDOZO et al., 2009). A imobilização de uma enzima significa combinar a seletividade, a estabilidade e a cinética dessa enzima com as propriedades físicas e químicas do transportador em uma formulação especializada que tem como papel principal maximizar a estabilidade física e enzimática do biocatalisador (BASSO E SERBAN, 2019). Diversas técnicas e protocolos já foram descritos para imobilização de enzimas. A escolha do método e do suporte a ser empregado dependerá principalmente das características físico-químicas da enzima e das condições de uso nas quais a enzima imobilizada será submetida, É igualmente importante avaliar o tempo e os custos necessários para se viabilizar o método de imobilização escolhido, uma vez que tais parâmetros irão se refletir no processo e, portanto, nos custos do produto final (FERNANDES, LIMA e LOPES, 2010).

Bassa e Serban (2019) pontuaram as principais vantagens e desvantagens da utilização de enzimas imobilizadas em processos industriais. A imobilização de biocatalizadores oferece vantagens como a facilidade para reutilização do biocatalizador e custos reduzidos em etapas de "downstream", entretanto, esses processos apresentam algumas limitações, visto que, há uma diminuição na atividade enzimática do imobilizado em comparação a enzima livre, além de custos adicionais referentes as etapas de imobilização, como apresentado no Quadro 1. 
Quadro 1. Vantagens e desvantagens de enzimas imobilizadas em processos industriais.

\begin{tabular}{|c|c|}
\hline VANTAGENS & DESVANTAGENS \\
\hline $\begin{array}{l}\text { - Fácil separação do biocatalisador; } \\
\text { - Custos reduzidos de processamento } \\
\text { "downstream”; } \\
\text { - Uso múltiplo de biocatalisador } \\
\text { (reciclagem); } \\
\text { - Melhor estabilidade, especialmente em } \\
\text { relação a solventes orgânicos e } \\
\text { temperaturas mais altas; } \\
\text { - Uso de reatores de leito fixo ou } \\
\text { batelada sem necessidade de } \\
\text { membrana para isolar enzima do } \\
\text { produto; } \\
\text { É possível co-imobilizar com outras } \\
\text { enzimas. }\end{array}$ & $\begin{array}{l}\text { - } \begin{array}{l}\text { Menor atividade enzimática em } \\
\text { comparação com a enzima nativa; }\end{array} \\
\text { - } \begin{array}{l}\text { Custos adicionais para transportadoras } \\
\text { e imobilização; }\end{array} \\
\text { - Taxas de reação mais baixas em } \\
\text { comparação com enzimas nativas; } \\
\text { - Sujeito a incrustação; } \\
\text { - Eliminação de enzima imobilizada } \\
\text { exaurida (incineração). }\end{array}$ \\
\hline
\end{tabular}

Fonte: Basso e Serban (2019)

As técnicas de imobilização de enzimas podem ser divididas em métodos químicos e físicos (Figura 1). Os métodos químicos envolvem a formação de ligações covalentes obtidas pela ligação de éter, tio-éter, amida ou carbamato entre a enzima e o suporte ou ainda através de ligações cruzadas entre a enzima e um agente reticulante. Nos métodos físicos, a enzima quando se liga por adsorção, formando ligações mais fracas com o suporte de imobilização (ligações de hidrogênio, ligação por afinidade, forças de Van der Waals, interações hidrofóbicas e ligação iônica), ou ainda podem ser aprisionadas ou encapsuladas (FURLANI et al., 2020). As interações enzima-suporte na imobilização podem ser vistas na Figura 1.

O uso de nanopartículas magnéticas como suporte sólido atribui ao biocatalisador um dos requisitos essenciais de fácil recuperação por simples aplicação de um campo magnético externo, reduzindo os custos de energia e tempo das etapas de centrifugação. Além do que nanomateriais apresentam uma alta relação superfície/volume facilitando assim a transferência de massa (PEREIRA et al., 2015). 
Figura 1. Classificação e representação de diferentes técnicas de imobilização de enzimas.

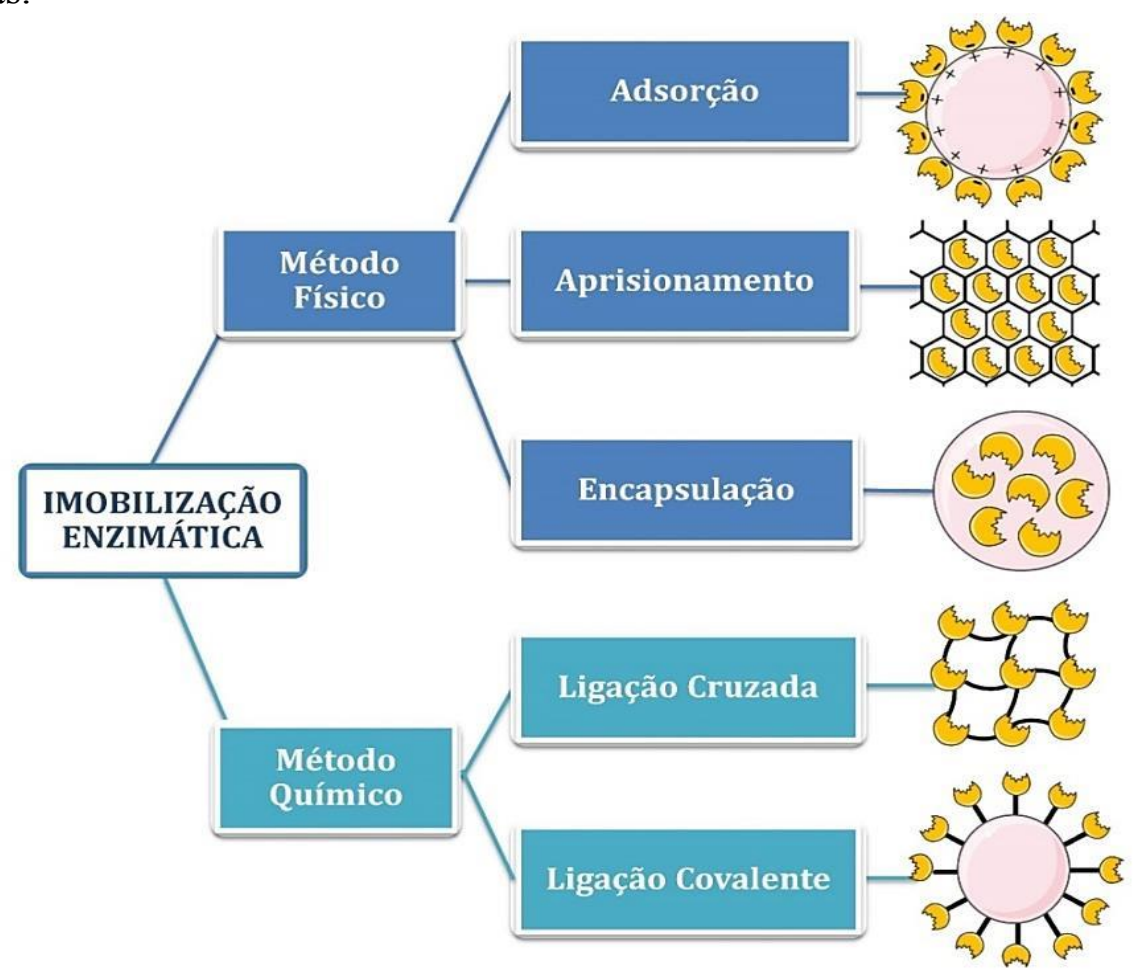

Fonte: Adaptado de Furlani et al., 2020; Souza et al., 2017.

\section{Imobilização enzimática em Nanopartículas magnéticas (NPMs)}

Nanopartículas magnéticas (NPMs) podem ser preparadas a partir de materiais de magnetização altamente saturados, tais como os metais de transição Ferro, Cobalto, Níquel e os óxidos metálicos $\mathrm{Fe}_{3} \mathrm{O}_{4}, \gamma-\mathrm{Fe}_{2} \mathrm{O}_{3}$, de acordo com vários métodos diferentes. Metais puros, como nanopartículas de ferro, possuem a maior magnetização, entretanto, também possuem alta toxicidade e são propensos à oxidação. Óxidos de metal mais estáveis e biocompatíveis, como nanopartículas são os de óxido de ferro superparamagnético, sendo preferidos, apesar de sua menor magnetização (QUAN et al., 2020). NPMs à base de óxido de ferro são mais comumente utilizadas e podem ser sintetizadas a partir de uma variedade de métodos químicos como síntese por decomposição térmica, reações hidrotermais, microemulsão e coprecipitação (FRANCISQUINI, SCHOENMAKER e SOUZA, 2010).

NPMs possuem propriedades intrínsecas interessantes para aplicações biotecnológicas, tais como: tamanho reduzido, área de superfície alta, resistência a altas temperaturas, altas interações enzimas-substrato e alta reatividade química atraindo a atenção dos cientistas. Além do que, devido à propriedade do superparamagnetismo é possível a recuperação do biocatalisador apenas por aplicação de um campo magnético ocasionando baixo estresse mecânico as enzimas (HU et al., 2009; COSTA et al., 2016). 
Embora as nanopartículas magnéticas possuam alta biocompatibilidade, mesmo sem modificação de superfície (QUAN et al., 2020). Comumente a superfície das nanopartículas é modificada visando facilitar o processo de imobilização enzimática, e contornar dificuldades como a distribuição não uniforme do tamanho da partícula e a fraca dispersibilidade aquosa, visto que, comportamentos restritos de nanomateriais em diferentes solventes limitam suas aplicações e, portanto, a modificação da superfície é uma estratégia importante que ajusta as propriedades dos nanomateriais para estender suas aplicações. A modificação da superfície pode alterar a propriedade existente ou pode introduzir propriedades completamente novas a partícula (SEENUVASAN et al., 2020).

A superfície das nanoestruturas pode ser modificada com materiais orgânicos (por exemplo, ácido láurico) e inorgânicos (por exemplo, $\mathrm{SiO}_{2}$ ) com o intuito de produzir partículas funcionalizadas (por exemplo, grupo amino) para imobilização enzimática (COSTA et al., 2016). Segundo Liu et al. (2020), a funcionalização da superfície das NPMs por compostos orgânicos é um método eficaz para aumentar a estabilidade das partículas durante o armazenamento e utilização. Além disso, as nanopartículas podem ser modificadas com certas biomoléculas para aumentar sua biocompatibilidade e melhor aplicação "in vivo", tornando-as mais específicas e expandindo o escopo de suas aplicações.

As estruturas das NPMs revestidas com compostos orgânicos podem ser divididas em núcleo-concha, matriz e concha-núcleo-concha (Figura 2), sendo formada por duas partes: um núcleo, geralmente formado por qualquer tipo de nanopartícula de óxido ferromagnético, classificado como magnetita, e uma casca/concha que pode ser um polímero, biopolímero ou biomolécula. As partículas modificadas por compostos orgânicos não só possuem as propriedades magnéticas básicas das NPMs, mas também apresentam boa biocompatibilidade e biodegradabilidade. Dentre os revestimentos inorgânicos a sílica é o material mais utilizado, devido a suas características inertes, com alta biocompatibilidade e baixa toxicidade, a sílica é um revestimento funcional para NPMs ideal para utilização no campo médico, pois permite a formação várias ligações de reticulação, além de, formar uma camada de proteção externa inerte para proteger as nanopartículas magnéticas internas (LIU et al., 2020). 
Figura 2. Estrutura principal das nanopartículas magnéticas modificadas por materiais orgânicos

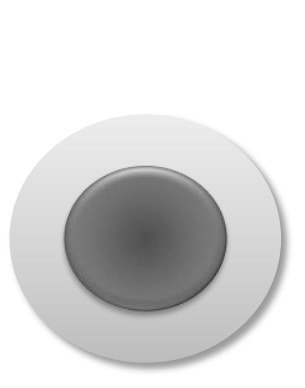

núcleo-concha

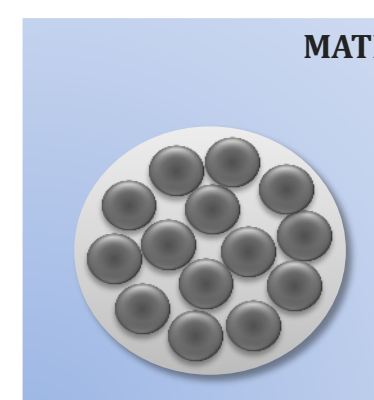

mosaico

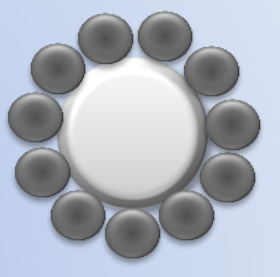

concha-núcleo

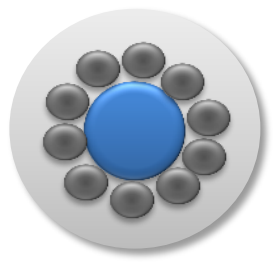

concha-núcleo-concha

Fonte: Adaptado de Liu et al., 2020

\section{Discussão}

A metodologia utilizada para a realização deste trabalho foi uma breve revisão bibliográfica, baseada em pesquisas de artigos científicos disponíveis nos bancos de dados: $\quad$ Scopus (http://www.scopus.com/); Science Direct (http://www.sciencedirect.com/), ISI Web of Science (http: //apps.isiknowledge.com) e PubMed (http://www.ncbi.nlm.nih.gov/pubmed), para a busca foi utilizado o seguinte termo de busca: ("magnetic nanoparticles") AND (coatings) AND ("enzyme immobilization”). Todo referencial teórico citado nesse artigo é meramente qualitativo sem nenhuma pretensão quantitativa.

Após a preparação das NPMs, algumas modificações devem ser realizadas para protegê-las contra agregações, oxidação e/ou alterações das cargas superfíciais, bem como, melhorar a capacidade de imobilização (DARWESH et al., 2019). A literatura traz vários estudos relacionados às NPMs recobertas com diversos materiais. Os materiais orgânicos possibilitam a preservação das características magnéticas das nanopartículas, biocompatibilidade, biodegradabilidade, além de fornecer grupos funcionais reativos (carboxila, amino, aldeído etc.) (LAURENT, et al. 2008). Exemplos desses materiais são moléculas pequenas e surfactantes, como ácido oleico, ácido cítrico, fosfanatos, aminoácidos e silanos; polímeros naturais e sintéticos, como dextran, quitosana, alginato, poli(etilenoglicol), poli(metilmetacrilato); e biomoléculas, como proteínas e anticorpos. Os materiais inorgânicos também são biocompatíveis e providenciam às nanopartículas estabilização, funcionalização e antioxidação. Pode-se citar como exemplos a sílica; metais e não-metais (ouro, prata, carbono); e óxidos metálicos e sulfetos ( $\mathrm{ZnO}, \mathrm{CaO}, \mathrm{A} 12 \mathrm{O} 3, \mathrm{ZnS})$ (TOMBÁCZ et al., 2015). A Tabela 1 mostra diferentes abordagens do uso de materiais para imobilização de enzimas. 
SILVA, M. C. T. S; FERNANDES, L. M. G; PORTO, A. L. F; CUNHA, M. N. C; PORTO, T. S.

Tabela 1. Tipos de revestimento em nanopartículas magnéticas para imobilização de diferentes enzimas.

\begin{tabular}{|c|c|c|c|c|c|}
\hline$\overline{\mathrm{ANPMs}}$ & $\begin{array}{l}\text { Revestimento } \\
\text { Orgânico / inorgânico }\end{array}$ & Enzima & $\overline{{ }^{\mathbf{B}} \mathbf{C R}}$ & $\begin{array}{l}{ }^{\mathrm{C} A R} \\
(\%) \\
\end{array}$ & Referências \\
\hline$\overline{\mathrm{Fe}_{3} \mathrm{O}_{4}}$ & Quitosana & Pectinase & 10 & $70,02 \%$ & "Nouri e Khodaiyan, 2020 \\
\hline $\mathrm{Fe}_{3} \mathrm{O}_{4}$ & ${ }^{\mathrm{D}} \mathrm{CMC}$ & NovoQ & 3 & $>95 \%$ & Ni et al., 2020 \\
\hline $\mathrm{Fe}_{3} \mathrm{O}_{4}$ & ${ }^{\mathrm{D}} \mathrm{CMC}$ & Lipase & 10 & $83,9 \%$ & Hongbo Suo et al., 2020 \\
\hline $\mathrm{Fe}_{3} \mathrm{O}_{4}$ & ${ }^{\mathrm{E}} \mathrm{CMD}$ & $\mathrm{ADH}$ & $* * *$ & $* * *$ & Vasić et al., 2020 \\
\hline $\mathrm{Fe}_{3} \mathrm{O}_{4}$ & FPoli(Catechol/TEPA) & Lipase & $* * *$ & $* * *$ & Tang et al., 2019 \\
\hline $\mathrm{Fe}_{3} \mathrm{O}_{4}$ & ${ }^{\mathrm{G}} \mathrm{CS}-\mathrm{TPP}$ & Alcalase & 10 & $60 \%$ & Chen et al., 2018 \\
\hline $\mathrm{Fe}_{3} \mathrm{O}_{4}$ & Lectinas ConA & Glicose oxidase & 8 & $99 \%$ & Yong et al., 2018 \\
\hline $\mathrm{Fe}_{3} \mathrm{O}_{4}$ & Alginato/quitosana & cloroperoxidase & 25 & $25 \%$ & García-Embid et al., 2018 \\
\hline $\mathrm{Fe}_{3} \mathrm{O}_{4}$ & Quitosana & Lipase & 20 & $83 \%$ & Mendes et al., 2011 \\
\hline $\mathrm{Fe}_{3} \mathrm{O}_{4}$ & ${ }^{\mathrm{H}} \mathrm{CT} / \mathrm{PDA}$ & $\alpha$-amilase & 6 & $70 \%$ & $\begin{array}{l}\text { Manthiriyappan e Lee, } \\
2011\end{array}$ \\
\hline $\mathrm{Fe}_{3} \mathrm{O}_{4}$ & Sílica/epoxi & Lipase & 7 & $79 \%$ & Afzal et al., 2020 \\
\hline $\mathrm{Fe}_{3} \mathrm{O}_{4}$ & IPEG- triclorotriazina & Pectinase & 10 & $55 \%$ & Kharazmi et al, 2020 \\
\hline $\mathrm{Fe}_{3} \mathrm{O}_{4}$ & IPEG- triclorotriazina & Xilanase & 9 & $50 \%$ & Kharazmi et al., 2020 \\
\hline $\mathrm{Fe}_{3} \mathrm{O}_{4}$ & Álcool polivinílico & Tripsina & 8 & $56 \%$ & Sahin e Ozmen, 2020 \\
\hline AuM & sílica mesoporosa & Celulase & 9 & $58 \%$ & Poorakbar et al., 2018 \\
\hline $\mathrm{Fe}_{3} \mathrm{O}_{4}$ & Sílica/naringina & $\alpha$-amilase & 10 & $50 \%$ & Defaei et al., 2018 \\
\hline $\mathrm{Fe}_{3} \mathrm{O}_{4}$ & Sílica/epoxi & L-Asparaginase & 10 & $65 \%$ & Ulu et al., 2018 \\
\hline $\mathrm{Fe}_{3} \mathrm{O}_{4}$ & JPAMAM & $\alpha$-glucosidase & $* * *$ & $* * *$ & Jiang et al., 2019 \\
\hline $\mathrm{Fe}_{3} \mathrm{O}_{4}$ & Sílica & Lacase & 6 & $75 \%$ & Fortes et al., 2017 \\
\hline $\mathrm{Fe}_{3} \mathrm{O}_{4}$ & ${ }^{\mathrm{K}} \mathrm{PANI}$ & Protease & 5 & $50 \%$ & Neto et al., 2017 \\
\hline $\mathrm{Fe}_{3} \mathrm{O}_{4}$ & LPUU & Lipase & $* * *$ & $* * *$ & Chiaradia et al., 2016 \\
\hline $\mathrm{Fe}_{3} \mathrm{O}_{4}$ & ${ }^{\mathrm{M}} \mathrm{PEIP}$ & Tripsina & 21 & $>90 \%$ & Monteil et al., 2014 \\
\hline $\mathrm{Fe}_{3} \mathrm{O}_{4}$ & Sílica & Glicose oxidase & 12 & $90 \%$ & Ashtari et al., 2012 \\
\hline
\end{tabular}

\footnotetext{
ANPMs - Nanopartículas magnéticas

${ }^{\mathrm{B}} \mathrm{CR}$ - Número de ciclos de reuso

${ }^{\mathrm{C}} \mathrm{AR}(\%)$ - Atividade residual após os ciclos de reuso

D NPM-CMC-Nanopartículas magnéticas de carboximetilcelulose

${ }^{\mathrm{E}} \mathrm{CMD}$ - Carboximetil dextrose

F Poli (Catecol/TEPA) - Catecol/tetraetileno pentamina

${ }^{\mathrm{G}}$ CS-TPP - Quitosana+tripolifosfato de sódio

${ }^{\mathrm{H}} \mathrm{CT} / \mathrm{PDA}$-quitina e polidopamina

${ }^{\text {I }}$ PEG - Polietilenoglicol

${ }^{\mathrm{J}}$ PAMAM - poliamidoamina

K PANI - polianilina

L PUU - poli(ureia-uretano)

M PEIP - polietilenimina parcialmente fosfonada

*** - Dados não apresentados
} 


\section{Tipos de revestimentos utilizados em nanopartículas magnéticas}

\section{Quitosana}

A quitosana é amplamente utilizada como suporte para a imobilização enzimática devido às suas diferentes configurações geométricas, tais como pós, flocos, hidrogéis, membranas, fibras e outros, e a presença de grupos hidroxila e aminoácidos (KUO et al., 2012). Devido à sua versatilidade, as partículas de quitosana também podem ser utilizadas como revestimento para nanopartículas magnéticas, como descrito por Mendes et al. (2011) quando utilizaram nanopartículas magnéticas de $\mathrm{Fe}_{3} \mathrm{O}_{4}$ revestidas com quitosana para a imobilização covalente de lipase de Candida rugosa. A lipase imobilizada apresentou melhor estabilidade operacional a maiores faixas térmicas e de $\mathrm{pH}$, permanecendo estável após 13 dias de armazenamento a $25^{\circ} \mathrm{C}$ com atividade residual de $83 \%$ após 20 ciclos.

NPMs revestidas com quitosana utilizando polissacarídeo de kefiran como agente de reticulação foram utilizadas na imobilização da enzima pectinase. Os resultados obtidos mostraram uma recuperação máxima da atividade da enzima pectinase imobilizada e após um mês, a atividade remanescente da enzima imobilizada foi de 60,23\%. A pectinase imobilizada manteve sua atividade em 70,02\% após 10 ciclos. A utilização nanopartículas magnéticas de quitosana em combinação com o reticulador poli-aldeído kefiran foi um método eficiente para imobilização de pectinases (NOURI e KHODAIYAN, 2020).

Resultados que corroboram com os apresentados por Chen et al. (2018) quando utilizaram partículas magnéticas $\mathrm{Fe}_{3} \mathrm{O}_{4}$ revestidas com quitosana e tripolifosfato de sódio $\left(\mathrm{Fe}_{3} \mathrm{O}_{4}\right.$-CS-TPP) para imobilização simultânea de alcalase e tripsina. Os NPMs de $\mathrm{Fe}_{3} \mathrm{O}_{4}$ - CS-TPP foram mais estáveis do que a enzima livre em temperaturas acima $40{ }^{\circ} \mathrm{C}$. A atividade residual do imobilizado foi preservada em $86 \%$ após 35 dias de armazenamento e reteve mais de $60 \%$ de sua atividade inicial após dez vezes de reutilização sucessiva. Resultados que confirmam a potencialidade da quitosana como revestimento de NPMs para imobilização de diferentes enzimas.

Uma abordagem diferente foi utilizada por Manthiriyappan e Lee (2011) quando utilizaram a quitina como matriz protetora e dispersiva para a preparação de partículas magnéticas pelo processo de co-precipitação. Posteriormente as partículas de quitina magnética (MCT) foram modificadas com dopamina para ser utilizada como uma matriz de imobilização enzimática eficaz, oferecendo superfície aderente para enzimas. Os resultados obtidos pelos autores para a imobilização da $\alpha$-amilase demonstraram uma taxa de hidrólise de amido comparável à da enzima livre. No entanto, as atividades relativas foram maiores do que a enzima livre em faixas mais amplas de $\mathrm{pH}$ e 
temperatura. Além disso, $\alpha$-amilase imobilizada reteve mais de $70 \%$ de sua atividade original após seis vezes de uso repetido.

Várias composições de nanopartículas de óxido de ferro revestidas com polidopamina e com uma concha polissacarídica obtidas através da combinação de quitosana e alginato foram usadas para imobilizar cloroperoxidase. A enzima foi imobilizada com sucesso com uma eficiência de aprisionamento entre $92 \%$ e $100 \%$ no caso de suportes com quitosana no interior e exterior respectivamente. Uma excelente estabilidade química foi observada no caso de um invólucro interno de alginato e um revestimento externo de quitosana, juntamente com uma excelente reutilização da enzima imobilizada, que foi reciclada para catalisar até 25 ciclos de reação consecutivos (GARCÍA-EMBID et al., 2018).

\section{Carboximetilcelulose (NPM-CMC) e Carboximetil-dextrana (CMD)}

Suo et al. (2020) sintetizaram nanopartículas magnéticas de carboximetilcelulose modificadas com líquidos iônicos e utilizaram como suporte para a imobilização enzimática. A enzima lipase foi utilizada como modelo e sua atividade específica imobilizada foi 1,43 vezes maior do que a atividade da enzima livre. Segundo os autores os compósitos desenvolvidos podem ser utilizados como excelentes suportes para imobilização de enzimas para aplicações industriais. Os resultados para testes de redutibilidade da enzima imobilizada mostraram que após 10 ciclos de reuso a enzima reteve $83,9 \%$ de sua atividade inicial. Os autores atribuem essa alta atividade ao suporte magnético que facilita a separação da enzima imobilizada, reduzindo perdas durante o processo de separação.

NPMs-CMC foram utilizadas para imobilizar a enzima pheniltransferase NovQ aplicada para a produção de vitamina $\mathrm{K}$ usando menadiona hidroquinol e dimetilalil difosfato (DMAPP) como substratos. Foi observado que carboximetilcelulose (CMC) e $\mathrm{Fe}_{3} \mathrm{O}_{4}$ formaram uma estrutura núcleo-concha com alta afinidade para a enzima imobilizada, sendo um suporte promissor em bioprocesso de produção de vitamina $\mathrm{K}$ (NI et al., 2020).

Carboximetil dextrana (CMD) foi covalentemente ligado a NPMs e a influência de diferentes concentrações nas características de nanopartículas magnéticas revestidas com CMD (CMD- NPMs) foi estudada. Os CMD3- NPMs preparados foram usados como transportadores para a imobilização da enzima álcool desidrogenase (ADH). As CMD- NPMs que formam sintetizados, exibiram uma camada de revestimento CMD que forneceu propriedades magnéticas e estruturais adequadas e, portanto, podem ser 
funcionalizados e usados na imobilização de compostos bioativos, como o ADH (VASIĆ et al., 2020).

\section{Naringina}

A naringina (4,5,7-trihidroxi flavanona-7-ramnoglucosídeo) é um flavonoide amargo presente em várias frutas cítricas. Naringina é uma biomolécula natural de baixo custo e hidrofílica, biocompatível e acessível, características que justificam seu uso na funcionalização da superfície de nanopartículas magnéticas.

A imobilização de enzimas em NPMs funcionalizados com naringina pode promover um novo microambiente vantajoso, incluindo a melhoria da estabilidade e do comportamento catalítico da enzima imobilizada. A imobilização da enzima $\alpha$-amilase em NPMs revestidas de sílica e funcionalizadas com naringina via interações iônicas. Os resultados mostraram que as condições ideais para imobilização da $\alpha$-amilase no nanocarreador sintetizado ocorreram em $\mathrm{pH} 6,5$ e $55^{\circ} \mathrm{C}$.

Os experimentos de reutilização revelaram manutenção de alta atividade da $\alpha$ amilase imobilizada mesmo após 10 ciclos de reação. A estabilidade de armazenamento da enzima imobilizada melhorou em comparação com a enzima livre, mantendo $60 \%$ de sua atividade inicial após 6 semanas de armazenamento. As melhorias nas propriedades catalíticas da enzima via imobilização tornaram este nanobiocatalisador um bom candidato em aplicações bioindustriais (DEFAEI et al., 2018).

\section{Lectina}

Nanopartículas magnéticas foram revestidas com lectinas ConA (NPMConA) para imobilização da enzima glicose oxidase (GO). A distorção conformacional mínima da enzima foi alcançada com NPMs revestido pela ConA, visto que pequenos desvios no espectro de dicroísmo circular (CD) são observados em comparação com aqueles sem ConA. Em comparação com as enzimas de difusão livre, as enzimas GOx-CAT imobilizadas em ConA-NPMs resultam em atividade aumentada de 1,3 vezes, apresentando maior estabilidade, além da facilidade de reciclagem e operação garantida pelo NPMs (YOUNG et al., 2018).

\section{Catecol/poliamida (CPA)}

Nanopartículas magnéticas revestidas por um sistema binário Catecol/ tetraetileno pentamina (CPA) barato e introduzindo braços espaçadores para ligação enzimática foram sintetizadas por Tang et al. (2019). Posteriormente, os autores utilizaram a imobilização da enzima lipase como modelo do processo de imobilização. 
A lipase foi imobilizada na superfície de tais materiais magnéticos funcionalizados com amino-epóxi através de troca iônica e fixação covalente com suporte com uma capacidade de carga de $180,6 \mathrm{mg} / \mathrm{g}$ e 69,2\% de recuperação da atividade enzimática em condições otimizadas. Além disso, a lipase imobilizada exibiu a faixa de tolerância melhorada de $\mathrm{pH}$, temperatura e estabilidade de armazenamento, bem como excelente capacidade de reutilização.

\section{Sílica}

A nanoestrutura da sílica ganhou atenção na área biomédica, devido à sua química bem definida, que permite a modificação da sua estrutura pelos grupos amina, carboxila e tiol, metacrilatos, enzimas, proteínas e DNA (LIMA, 2016).

A lacase, uma enzima oxidativa com inúmeras aplicações industriais, requer novas tecnologias para sua imobilização a fim de melhorar sua atividade biocatalítica com custos reduzidos. Fortes et al. (2017) avaliaram a imobilização desta enzima em NPMs revestidas com sílica, nas condições ideais, a maior atividade de recuperação da lacase imobilizada atingiu 36,3 U/L, e apresentou atividade residual acima de 75\% após 6 ciclos consecutivos de reação. Além disto, a estabilidade térmica da lacase imobilizada foi melhorada em comparação a enzima livre.

Resultados semelhantes foram obtidos por Ashtari et al. (2012) na imobilização da glicose oxidase (GOD) em NPMs encapsuladas em sílica preparadas através do método de microemulsão. As NPMs obtidas não apresentaram qualquer atividade citotóxica contra células de carcinoma de pulmão humano e letalidade de salmoura. A ligação da enzima ao suporte foi confirmada pelos espectros de FTIR. O estudo da estabilidade de armazenamento mostrou que a glicose oxidase imobilizada reteve $98 \%$ de sua atividade inicial após 45 dias e 90\% da atividade também permaneceu após 12 usos repetidos. Melhorias consideráveis na estabilidade térmica da enzima imobilizada também foram observadas em temperaturas elevadas de até $80^{\circ} \mathrm{C}$, além disto, a atividade da enzima imobilizada foi menos sensível às mudanças de $\mathrm{pH}$ da solução.

Ulu et al. (2018) avaliou a imobilização de L-Asparaginase em NPMs revestidas com sílicas e funcionalizadas por organossilano (epóxi). A L-Asparaginase imobilizada apresentou maior atividade em valores elevados de $\mathrm{pH}$ e temperatura. Também manteve mais de $92 \%$ da atividade inicial após incubação a $55{ }^{\circ} \mathrm{C}$ por $3 \mathrm{~h}$. Em relação aos valores cinéticos, a L-Asparaginase imobilizada apresentou maior $\mathrm{V}_{\max }$ e $\mathrm{K}_{\mathrm{m}}$ menor em comparação à enzima nativa. Além disso, apresentou excelente reutilização por 12 ciclos sucessivos. Após 30 dias de armazenamento a $4{ }^{\circ} \mathrm{C}$ e $25{ }^{\circ} \mathrm{C}$, a L-Asparaginase imobilizada reteve $54 \%$ e $26 \%$ de suas atividades iniciais, enquanto a L-Asparaginase 
nativa perdeu cerca de $68 \%$ e $84 \%$ de sua atividade inicial, respectivamente. NPMs semelhantes foram utilizadas com sucesso na imobilização de lipases (AFZAL et al., 2019).

Poorakbar et al. (2018) utilizou nanopartículas magnéticas de ouro (AuM) revestidas com sílica mesoporosa para imobilização de celulase por ligação covalente com uma eficiência de ligação em 76\%. A enzima imobilizada manteve 58\% de sua atividade catalítica inicial após nove horas. Nesta pesquisa, um novo nanossistema foi desenhado como um suporte sólido para a imobilização da celulase, aumentando sua estabilidade térmica e facilitou seu armazenamento. Além disso, a enzima imobilizada pode ser aplicada em uma faixa mais ampla de temperatura e pH em comparação com a enzima livre, com separação da enzima de forma simples realizada por um ímã externo.

\section{Polietilenimina (PEIP)}

A polietilenimina parcialmente fosfonada (PEIP) foi desenvolvida como um agente de revestimento facilmente funcionalizável para nanopartículas de óxido de ferro. A imobilização por tripsina foi realizada como modelo, os resultados obtidos evidenciaram que o PEIP contribuiu para a alta estabilidade do material, por meio de uma forte ligação covalente com o grupo amino da enzima. A resistência à hidrólise e ao aumento da temperatura garantiram a obtenção de um nanomaterial magnético altamente reciclável projetado para análises proteômicas. Podendo servir para suporte de numerosas enzimas (MONTEIL et al., 2014).

\section{Álcool polivinílico}

Sahin e Ozmen (2020) avaliaram nanopartículas magnéticas revestidas com álcool polivinílico e ativadas com glutaraldeído para imobilização de tripsina. A enzima imobilizada foi mais estável do que a enzima livre a $40{ }^{\circ} \mathrm{C}$ e reteve $50 \%$ de sua atividade inicial, após 12 dias a $4{ }^{\circ} \mathrm{C}$. Apresentando atividade residual de $56 \%$ após oito reutilizações sucessivas. O desempenho da enzima imobilizada foi avaliado pela digestão do citocromo $\mathrm{C}$, fragmentos de peptídeo em solução digerida foram determinados usando espectrometria de massa MALDI-TOF. A tripsina imobilizada apresentou atividade proteolítica efetiva em menor tempo $(15 \mathrm{~min})$ do que a tripsina livre (24 h). Portanto, é possível inferir que a imobilização enzimática nas condições descritas pode ser um bioprocesso promissor para estudos de proteômica em larga escala e aplicações práticas. 


\section{Polietileno glicol (PEG)}

NPMs revestidas com polietilenoglicol via triclorotriazina com alta eficiência de carregamento, foram utilizadas para a imobilização covalente da pectinase. A enzima imobilizada mostrou atividade catalítica aprimorada, estabilidade operacional e fácil reutilização. Estudos de estabilidade térmica e de $\mathrm{pH}$ mostraram um melhor desempenho da pectinase imobilizada em comparação a enzima livre. Além disso, a enzima imobilizada reteve até 55 e 94\% de sua atividade inicial após 10 reciclagens e 125 dias de armazenamento a $25{ }^{\circ} \mathrm{C}$, respectivamente. Além disso, a redução da turbidez ocorreu em até 59\% no suco de abacaxi tratado com pectinase imobilizada, sugerindo aplicabilidade desse sistema nas indústrias de sucos e alimentos (KHARAZMI et al. 2020). Da mesma forma, Kharazmi et al. (2020) utilizaram NPMs semelhantes na imobilização de xilanase estabilizada com atividade catalítica e estabilidade melhoradas. A enzima imobilizada foi aplicada em processos de clarificação de suco de abacaxi e notavelmente, reteve 50\% de sua atividade inicial após nove reciclagens de 120 min de incubação a $50{ }^{\circ} \mathrm{C}$ a pH 4,5.

\section{Polianilina (PANI)}

A polianilina (PANI) representa uma classe de polímeros altamente promissores, em função de seu baixo custo de síntese, facilidade operacional e por apresentar propriedade condutora de elétrons, pois, dependendo das condições de síntese, o polímero pode atuar como semicondutor. A protease de Penicillium aurantiogriseum URM4622 foi imobilizada em nanopartículas magnéticas revestidas com polianilina (PANI) ativadas com glutaraldeído. A protease foi covalentemente ligada às nanopartículas via glutaraldeído a $25{ }^{\circ} \mathrm{C}$. Foram determinadas as condições ótimas para imobilização enzimática e a caracterização da enzima imobilizada mostrou um comportamento ligeiramente diferente em comparação com a enzima livre. O processo de imobilização foi capaz de reutilizar a enzima até 5 vezes mantendo mais de $50 \%$ da sua atividade inicial. Os resultados obtidos comprovam que as nanopartículas ferromagnéticas revestidas com PANI são suporte eficiente para imobilização de protease fúngica (NETO et al., 2017).

\section{Poli (uréia-uretano) (PUU)}

Chiaradia et al. (2016) avaliaram a imobilização de lipase produzida por Candida antarctica, em nanopartículas magnéticas de poli (ureia-uretano) (NPMs PUU) em uma única etapa durante a polimerização em miniemulsão interfacial. Imagens de microscopia eletrônica de transmissão mostraram a morfologia de 
nanopartículas magnéticas sintetizadas encapsuladas em nanopartículas de poli (ureiauretano) e imagens de microscopia de fluorescência confirmaram a imobilização da enzima em NPMs -PUU. Após o processo de imobilização a lipase imobilizada foi atraída por um campo magnético externo e utilizada como biocatalisador para a síntese dos ésteres etil oleato, geranil propionato e geranil oleato. Conversões de ésteres acima de $85 \%$ foram obtidas para todos os sistemas com base nos conteúdos de ácidos graxos livres medidos por titulação até pH 11 confirmando a eficiência do processo de imobilização.

\section{Considerações Finais}

A imobilização de enzimas em nanopartículas magnéticas sem revestimento faz com que elas estejam altamente susceptíveis à oxidação, diminuindo sua resposta ao campo magnético. Como consequência, o suporte apresenta uma vida útil curta, diminuindo o potencial de reuso do biocatalisador. Ademais, o produto pode ser contaminado devido à perda de magnetização da amostra, gerando problemas no processo produtivo. Desta forma, é necessário realizar o revestimento da superfície dessas partículas com materiais que possuam características compatíveis com a enzima alvo e com o bioprocesso no qual ela será empregada, garantindo assim, uma maior estabilidade e eficiência catalítica. Requisitos estes de suma importância para uso em diversos setores industriais.

\section{Referências}

AFZAL, H. A., GHORPADE, R. V., THORVE, A. K., NAGARAJA, S., ALDHUBIAB, B. E., MERAVANIGE, G., ... \& ROOPASHREE, T. S. (2020). Epoxy functionalized polymer grafted magnetic nanoparticles by facile surface-initiated polymerization for immobilization studies of Candida Antarctica lipase B. Reactive and Functional Polymers, 147, 104454.

ASHTARI, K., KHAJEH, K., FASIHI, J., ASHTARI, P., RAMAZANI, A., \& VALI, H. (2012). Silica-encapsulated magnetic nanoparticles: enzyme immobilization and cytotoxic study. International journal of biological macromolecules, 50(4), 1063-1069.

BASSO, A., \& SERBAN, S. (2019). Industrial applications of immobilized enzymes: A review. Molecular Catalysis, 479, 110607.

BEDÊ, P. M., (2010). Produção e Caracterização de Nanopartículas Polimérico Magnéticas para Aplicações Biomédicas. Dissertação de M. Sc., Instituto Militarde Engenharia, Rio de Janeiro, RJ, Brasil.

CARDOSO, C. L., MORAES, M. C. D., \& CASS, Q. B. (2009). Imobilização de enzimas em suportes cromatográficos: uma ferramenta na busca por substâncias bioativas. Química Nova, 32(1), 175-187. 
CHEN, Z., WANG, X., CHEN, Y., XUE, Z., GUO, Q., MA, Q., \& CHEN, H. (2018). Preparation and characterization of a novel nanocomposite with double enzymes immobilized on magnetic $\mathrm{Fe} 3 \mathrm{O} 4$-chitosan-sodium tripolyphosphate. Colloids and Surfaces B: Biointerfaces, 169, 280-288.

CHIARADIA, V., VALÉRIO, A., DE OLIVEIRA, D., ARAÚJO, P. H. H., \& SAYER, C. (2016). Simultaneous single-step immobilization of Candida antarctica lipase B and incorporation of magnetic nanoparticles on poly(urea-urethane) nanoparticles by interfacial miniemulsion polymerization. Journal of Molecular Catalysis B: Enzymatic, $131,31-35$.

CHOI, J. M., HAN, S. S., \& KIM, H. S. (2015). Industrial applications of enzyme biocatalysis: Current status and future aspects. Biotechnology advances, 33(7), 14431454.

COSTA, V.M., SOUZA, M. C. FECHINE, M. P. B. A., MACEDO, A.C. \& GONÇALVES, L. R. B. (2016). Nanobiocatalytic systems based on Lipase-Fe3O4 and conventional systems for isoniazid synthesis: a comparative study, Brazilian Journal of Chemical Engineering, 33(3), 661-673.

DARWESH, O. M., ALI, S. S., MATTER, I. A., ELSAMAHY, T., \& MAHMOUD, Y. A. (2020). Enzymes immobilization onto magnetic nanoparticles to improve industrial and environmental applications. In Methods in Enzymology, Academic Press, 630, 481502.

DE OLIVEIRA, R. L., DIAS, J. L., DA SILVA, O. S., \& PORTO, T. S. (2018). Immobilization of pectinase from Aspergillus aculeatus in alginate beads and clarification of apple and umbu juices in a packed bed reactor. Food and bioproducts processing, 109, 9-18.

DEFAEI, M., TAHERI-KAFRANI, A., MIROLIAEI, M., \& YAGHMAEI, P. (2018). Improvement of stability and reusability of $\alpha$-amylase immobilized on naringin functionalized magnetic nanoparticles: A robust nanobiocatalyst. International Journal of Biological Macromolecules, 113, 354-360.

FERNANDES, K. F., LIMA, C. S., \& LOPES, F. M. (2010). Técnicas de imobilização de enzimas. Revista Processos Químicos, 4(7), 53-58.

FERNANDES, L. M. G., DA CUNHA, M. N. C., DE CARVALHO SILVA, J., PORTO, A. L. F., \& PORTO, T. S. (2020). Purification and characterization of a novel Aspergillus heteromorphus URM 0269 protease extracted by aqueous two-phase systems PEG/citrate. Journal of Molecular Liquids, 113957.

FERRARA, M. A. Cap. 6 Bioprocessos para produção de enzimas. FORTES, C. C. S., SILVA, A. L. D., XAVIER, A. M. R. B., \& TAVARES, A. P. M. (2017). Optimization of enzyme immobilization on functionalized magnetic nanoparticles for laccase biocatalytic reactions. Chemical Engineering and Processing: Process Intensification, $117,1-8$.

FRANCISQUINI, E., SCHOENMAKER, J. \& SOUZA, J. A. (2014) Nanopartículas Magnéticas e suas Aplicações. In: (Ed.). Série química: Ciência e tecnologia-química supramolecular e nanotecnologia. São Paulo: Atheneu, 592p. 
FURLANI, I. L., AMARAL, B. S., OLIVEIRA, R. V., \& CASS, Q. B. (2020). Imobilização enzimática: conceito e efeitos na proteólise. Química Nova, 43(4), 463473.

GARCÍA-EMBID, S., DI RENZO, F., DE MATTEIS, L., SPRETI, N., \& M. DE LA FUENTE, J. (2018). Magnetic separation and high reusability of chloroperoxidase entrapped in multi polysaccharide micro-supports. Applied Catalysis A: General, 560, 94-102.

GENNARI, A., FÜHR, A. J., VOLPATO, G., \& DE SOUZA, C. F. V. (2020). Magnetic cellulose: Versatile support for enzyme immobilization-A review. Carbohydrate Polymers, 116646.

HU, B.; PAN, J.; YU, H.; LIU, J. \& XU, J. (2009) Immobilization of Serratia marcescens lipase onto amino-functionalized magnetic nanoparticles for repeated use in enzymatic synthesis of Diltiazem intermediate. Process Biochemistry, Elsevier, 44(9), 1019-1024.

JIANG, J., YU, Y., WANG, L., LI, J., LING, J., LI, Y., \& DUAN, G. (2019). Enzyme immobilized on polyamidoamine-coated magnetic microspheres for $\alpha$-glucosidase inhibitors screening from Radix Paeoniae Rubra extracts accompanied with molecular modeling. Talanta. 195, 127-136.

KHARAZMI, S., TAHERI-KAFRANI, A., \& SOOZANIPOUR, A. (2020). Efficient immobilization of pectinase on trichlorotriazine-functionalized polyethylene glycolgrafted magnetic nanoparticles: A stable and robust nanobiocatalyst for fruit juice clarification. Food Chemistry, 126890.

KHARAZMI, S., TAHERI-KAFRANI, A., SOOZANIPOUR, A., NASROLLAHZADEH, M., \& VARMA, R. S. (2020). Xylanase immobilization onto trichlorotriazine-functionalized polyethylene glycol grafted magnetic nanoparticles: A thermostable and robust nanobiocatalyst for fruit juice clarification. International Journal of Biological Macromolecules.

KUO, C. H., LIU, Y. C., CHANG, C. M. J., CHEN, J. H., CHANG, C., \& SHIEH, C. J. (2012). Optimum conditions for lipase immobilization on chitosan-coated $\mathrm{Fe}_{3} \mathrm{O}_{4}$ nanoparticles. Carbohydrate Polymers, 87(4), 2538-2545.

LAURENT, S., FORGE, D., PORT, M., ROCH, A., ROBIC, C., VANDER ELST, L., \& MULLER, R. N. (2008). Magnetic iron oxide nanoparticles: synthesis, stabilization, vectorization, physicochemical characterizations, and biological applications. Chemical Reviews, 108(6), 2064-2110.

LIMA, J. S., ARAÚJO, P. H. H., SAYER, C., SOUZA, A. A. U., VIEGAS, A. C., \& DE OLIVEIRA, D. (2016). Cellulase immobilization on magnetic nanoparticles encapsulated in polymer nanospheres. Bioprocess and Biosystems Engineering, 40(4), 511-518.

LIU, S., YU, B., WANG, S., SHEN, Y., \& CONG, H. (2020). Preparation, surface functionalization and application of $\mathrm{Fe} 3 \mathrm{O} 4$ magnetic nanoparticles. Advances in Colloid and Interface Science, 102165. 
MENDES, A. A., OLIVEIRA, P. C.; CASTRO, H. F. \& GIORDANO, R L. C. (2011) Application of chitosan as support for immobilization of enzymes of industrial interest. Quím. Nova [online], 34(5), 831-840.

MONTEIL, C., BAR, N., RETOUX, R., HENRY, J., BERNAY, B., \& VILLEMIN, D. (2014). Partially phosphonated polyethylenimine-coated nanoparticles as convenient support for enzyme immobilization in bioprocessing. Sensors and Actuators B: Chemical, 192, 269-274.

NASCIMENTO, T. P., SAlES, A. E., PORTO, C. S., BRANDÃO, R. M. P., DE CAMPOS-TAKAKI, G. M., TEIXEIRA, J. A. C., ... \& CONVERTI, A. (2016). Purification of a fibrinolytic protease from Mucor subtilissimus UCP 1262 by aqueous two-phase systems (PEG/sulfate). Journal of Chromatography B, 1025, 16-24.

NETO, J. M. W. D., MACIEL, J. C., CAMPOS, J. F., CARVALHO JUNIOR, L. B., MARQUES, D. A. V., LIMA, C. A. \& PORTO, A. L. F. (2017) Optimization of Penicillium aurantiogriseum protease immobilization on magnetic nanoparticles for antioxidant peptides' obtainment, Preparative Biochemistry and Biotechnology, 47(7), 644-654

NI, W., ZHENG, Z., LIU, H., WANG, P., WANG, L., WANG, H., ...\& ZHAO, G. (2020). Synthesis of the carboxymethyl cellulose magnetic nanoparticles for efficient immobilization of prenyltransferase NovQ. Carbohydrate Polymers, 235, 115955. Falta

NOURI, M., \& FARAMARZ KHODAIYAN. (2020). Green synthesis of chitosan magnetic nanoparticles and their application with poly-aldehyde kefiran cross-linker to immobilize pectinase enzyme. Biocatalysis and Agricultural Biotechnology, 101681. Falta

PEREIRA, M.G., FACCHINI, F.D.A., FILÓ, L.E.C., POLIZELI, A.M., VICI, A.C., JORGE, J.A., FERNANDEZ-LORENTE, G., PESSELA, B.C., GUISAN, J.M. \& POLIZELI, M. L. T. M. . (2015). Immobilized lipase from Hypocrea pseudokoningii on hydrophobic and ionic supports: Determination of thermal and organic solvent stabilities for applications in the oleochemical industry, Process Biochemistry, 50(4), 561-570.

POORAKBAR, E., SHAFIEE, A., SABOURY, A. A., RAD, B. L., KHOSHNEVISAN, K., MA'MANI, L., ... \& HOSSEINI, M. (2018). Synthesis of magnetic gold mesoporous silica nanoparticles core shell for cellulase enzyme immobilization: Improvement of enzymatic activity and thermal stability. Process biochemistry, 71, 92-100.

QUAN K, ZHANG Z, REN Y, BUSSCHER HJ, VAN DER MEI HC \& PETERSON BW, (2020). Possibilities and impossibilities of magnetic nanoparticle use in the control of infectious biofilms, Journal of Materials Science \& Technology.

SAHIN, S., \& OZMEN, I. (2020). Covalent immobilization of trypsin on polyvinyl alcohol-coated magnetic nanoparticles activated with glutaraldehyde. Journal of Pharmaceutical and Biomedical Analysis, 184, 113195.

SEENUVASAN, M., KUMAR, K. S., KUMAR, A., \& PARTHIBAN, R. (2020). Review on surface modification of nanocarriers to overcome diffusion limitations: An enzyme immobilization aspect. Biochemical Engineering Journal, 107574. 
SINGH, R., MITTAL, A., KUMAR, M., \& MEHTA, PK (2016). Proteases microbianas em aplicações comerciais. J Pharm Chem Biol Sei, 4 (3), 365-74.

SOUZA, L. T., VERÍSSIMO, L. A., PESSELA, B. C., SANTORO, R. R., RESENDE, R. R., \& MENDES, A. A. (2017). Imobilização enzimática: princípios fundamentais e tipos de suporte, In: RESENDE, R. R. Biotecnologia Aplicada à Agro\&Indústria. Vol 4. Editora Blucher.

SUO, H., GAO, Z., XU, L., XU, C., YU, D., XIANG, X., ...\& HU, Y. (2020). Synthesis of functional ionic liquid modified magnetic chitosan nanoparticles for porcine pancreatic lipase immobilization. Materials Science and Engineering: C, 96, 356-364.

SURESHKUMAR, M., \& LEE, C.-K. (2011). Polydopamine coated magnetic-chitin (MCT) particles as a new matrix for enzyme immobilization. Carbohydrate Polymers, 84(2), 775-780

TANG, W., CHEN, C., SUN, W., WANG, P., \& WEI, D. (2019). Low-cost mussel inspired poly (Catechol/Polyamine) modified magnetic nanoparticles as a versatile platform for enhanced activity of immobilized enzyme. International journal of biological macromolecules, 128, 814-824.

TAVANO, O. L., BERENGUER- MURCIA, A., SECUNDO, F., \& FERNANDEZLAFUENTE, R. (2018). Biotechnological applications of proteases in food technology. Comprehensive reviews in food science and food safety, 17(2), 412-436.

TOMBÁCZ, E., TURCU, R., SOCOLIUC, V., \& VÉKÁS, L. (2015). Magnetic iron oxide nanoparticles: Recent trends in design and synthesis of magnetoresponsive nanosystems. Biochemical and Biophysical Research Communications, 468(3), 442453.

TÜZMEN N, KALBURCU T. \& DENIZLI A (2012) $\alpha$-Amylase immobilization onto dye attached magnetic beads: Optimization and characterization. Journal of Molecular Catalysis B: Enzymatic,78, 16-23.

ULU, A., OZCAN, I., KOYTEPE, S., \& ATES, B. (2018). Projeto de nanopartículas core-shell de $\mathrm{Fe}_{3} \mathrm{O}_{4}$ MCM-41 funcionalizadas com epóxi para imobilização enzimática. International Journal of Biological Macromolecules, 115, 1122-1130.

VASIĆ, K., KNEZ, Ž., KONSTANTINOVA, E. A., KOKORIN, A. I., GYERGYEK, S., \& LEITGEB, M. (2020). Structural and magnetic characteristics of carboxymethyl dextran coated magnetic nanoparticles: From characterization to immobilization application. Reactive and Functional Polymers, 148, 104481.

WU, W., HE, Q. \& JIANG, C. (2008). Magnetic iron oxide nanoparticles: Synthesis and surface functionalization strategies. Nanoscale Research Letters, 3(11), 397-415.

YONG, Y., SU, R., LIU, X., XU, W., ZHANG, Y., WANG, R., ...\& LIU, Z. (2018). Lectin corona enhances enzymatic catalysis on the surface of magnetic nanoparticles. Biochemical Engineering Journal, 129, 26-32. 